\title{
Antidepressant treatment of depression
} in real-world settings

EDITED BY J. MENDLEWICZ

sl Optimising antidepressant use in clinical practice: towards criteria for antidepressant selection

J. Mendlewicz

s4 Clinical importance of long-term antidepressant treatment

R. M. A. Hirschfeld

s9 Antidepressant use in clinical practice: efficacy $v$. effectiveness J. Donoghue and T. R. Hylan

sl8 Antidepressant selection and economic outcome: a review of methods and studies from clinical practice

W. H. Crown 